\title{
Experimental study of electron effects in heavy-ion beams
}

\section{•A.W. MOLVIKa,}

-With F.M. BIENIOSEK', D. Bacab , R.H. COHEN ${ }^{a}$, A. FRIEDMANa, M.A. FURMAN E.P. LEE ${ }^{b}$, S.M. LUND ${ }^{\mathrm{a}}$, L. PROST ${ }^{\mathrm{b}}$, P.A. SEIDL ${ }^{\mathrm{b}}$, J-L. VAY ${ }^{\mathrm{b}}$. a. LLNL b. LBNL

-Heavy-Ion Fusion Virtual National Laboratory HIF-VNL

\section{Presented to}

International workshop in physics of high energy density in matter, session on gas/electron cloud effects in high-ion-current accelerators

Waldemar-Petersen-Haus, Hirschegg, Austria

Feb. 2-7, 2003 


\section{Stray electron production is a concern in accelerators - but incompletely understood}

Proton Storage Ring
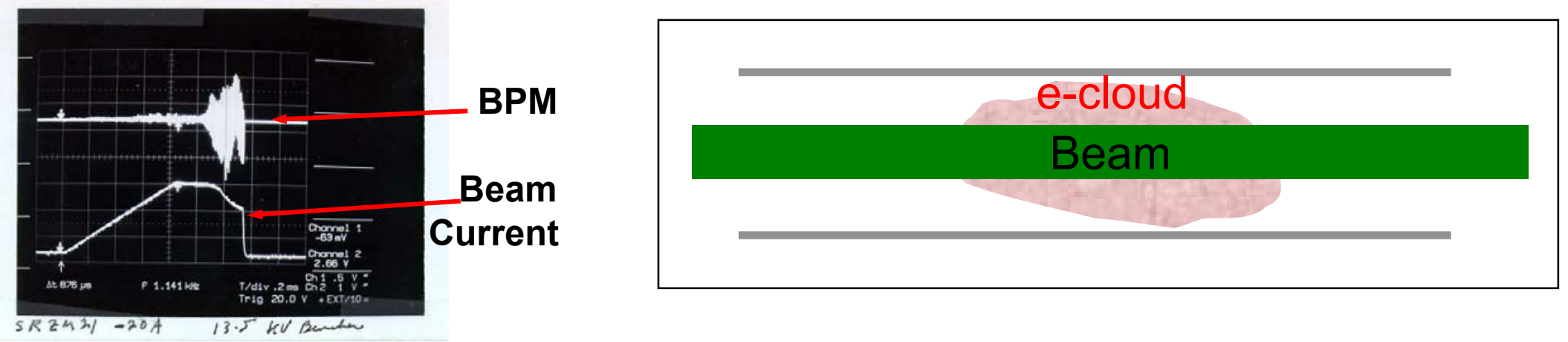

Electrons can be trapped in potential well of positive particle beam

-These effects limit present accelerators and put future accelerators at risk

-We are beginning coordinated experiments, theory, and simulations to understand phenomena and develop mitigating mechanisms to expand limits. 


\section{Beam hitting gas or walls creates electrons and gas - these can multiply}

Beam on gas, $I_{b}$

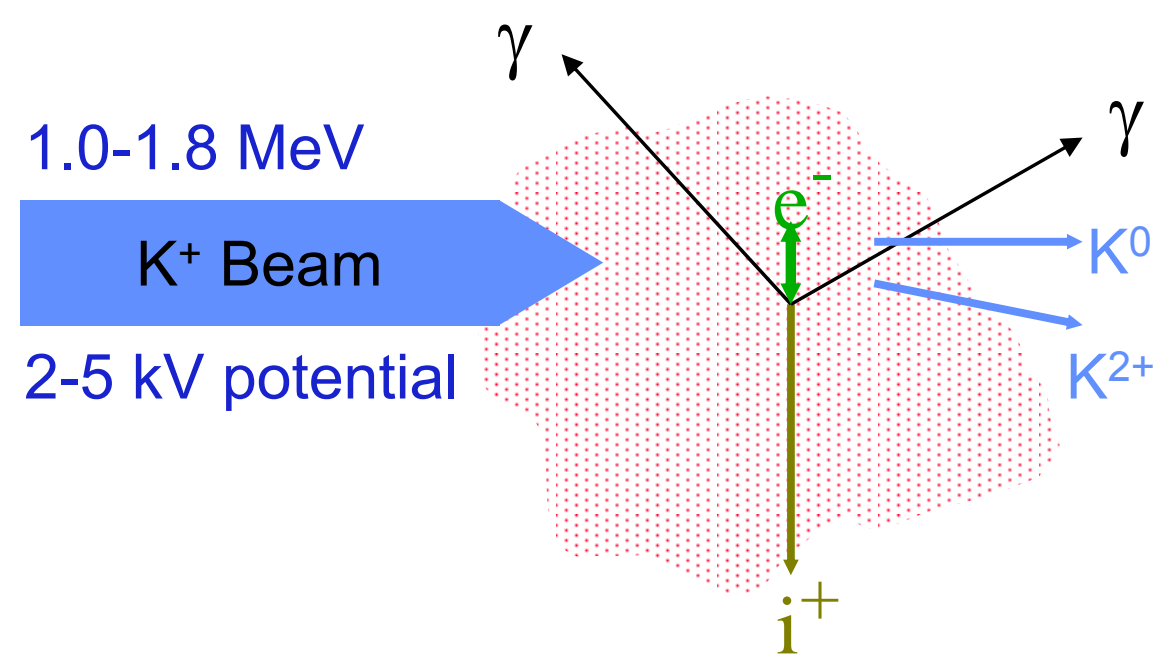

Beam loss to walls, $I_{b w}$

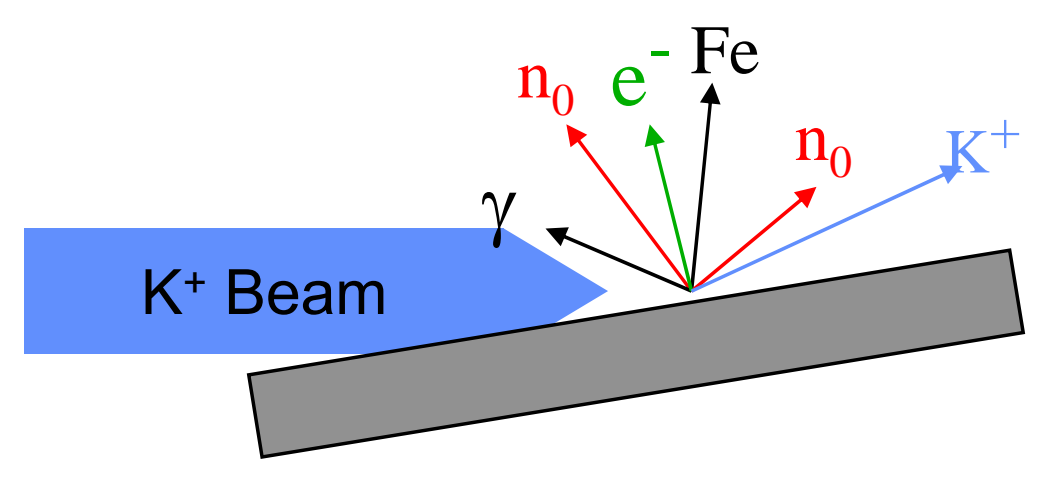

These interaction products create opportunities for diagnostics, but provide a hostile environment 


\section{TRIM Monte Carlo Code predicts significant beam ion scattering near grazing incidence}

- $60-70 \%$ scatter at $88-89^{\circ}$

- $0.05-0.5 \%$ scatter at $0-45^{\circ}$

- Scattering approaches $90^{\circ}(1.57$

rad), difficult to eliminate by

collimation.

- Issue - causes electron emission

$20001.8 \mathrm{MeV} \mathrm{K}+$ ions incident at 88 deg., $64 \%$ scatter

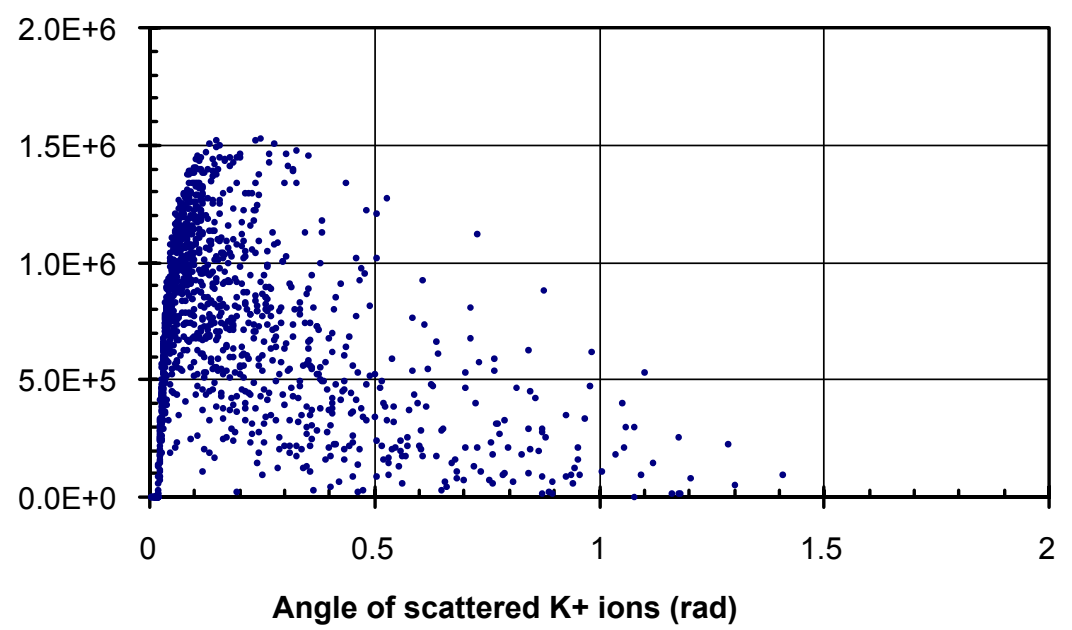

10,000 1.8 MeV K+ incident at 70 deg., $10 \%$ scatter

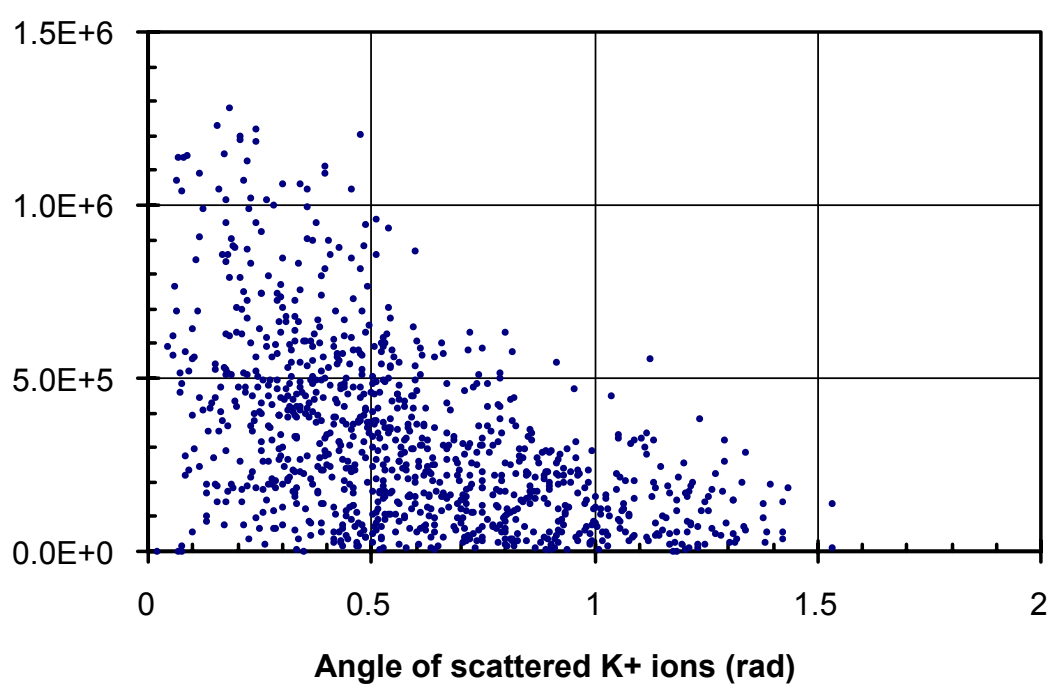

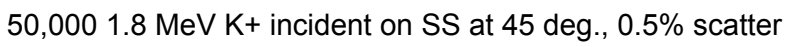

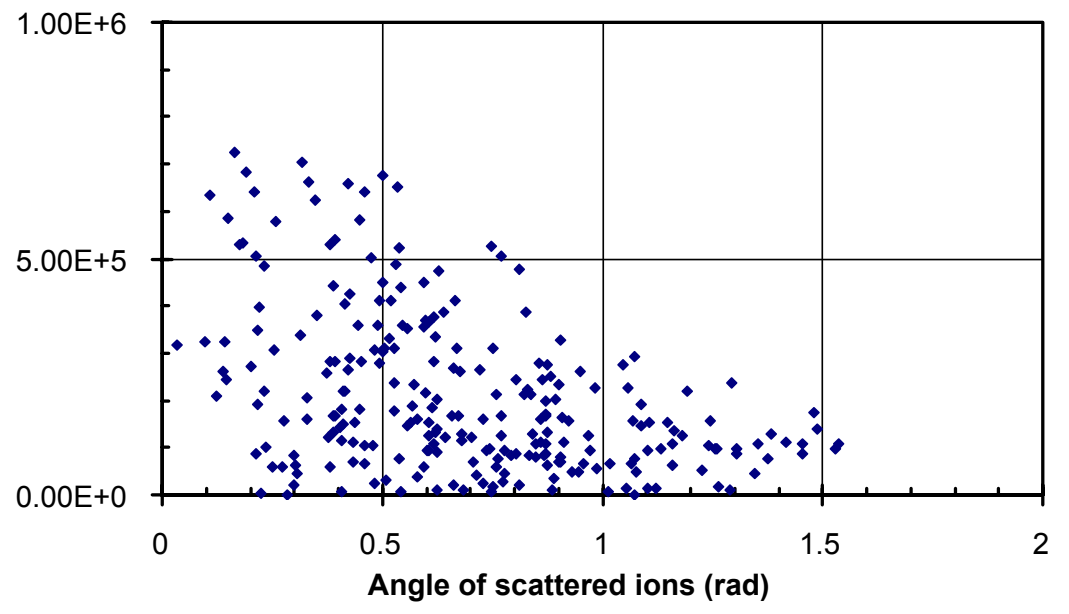




\section{Hostile environment for diagnostics}

\section{Beam loss to walls}

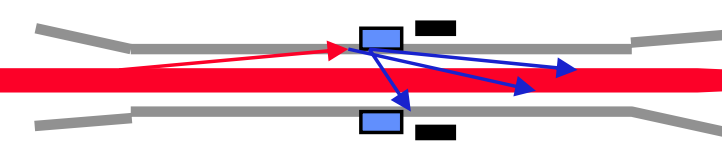

Scattered ions:
- Energetic ( $\geq 1 \mathrm{MeV}$ ) ions strike wall near grazing incidence, generate electron emission, gas desorption, and scattered beam ions.

-TRIM Monte Carlo Code predicts $\sim 70 \%$ reflection at $89^{\circ}$, at angles mostly $\leq 30^{\circ}$, but a few approaching $90^{\circ}$, with energies ranging from zero to near beam energy.

- These cause electron emission from walls

- Series of anti-reflection apertures in beam tube could reduce scattered ions by orders of magnitude.

Secondary electron suppression:

$\mathrm{B}$

Capacitive coupling from beam:

Signal to noise:
- Cannot suppress electrons with biased grids, when both grids and collector are impinged by scattered ions.

- Magnetic suppression in the lobes of magnetic quadrupole fields appears feasible as shown at left.

- Magnitude 500 times expelled ion current

- Shield with grids. Subtract remaining contribution.

- Signals as low as $\sim 1 \mu \mathrm{A}$, noise from spark-gaps - Shield, preamps near collectors. 


\section{Some simple diagnostics can measure electron related parameters in magnetic quadrupoles}

Flush probes:

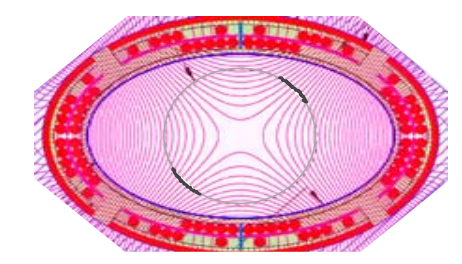

Capacitive probes:
- (Current/electron emission coefficient [from GESD]) = Halo loss + scattered ions to wall

- Signal $\propto \phi_{\mathbf{b}} \propto\left(\mathbf{n}_{\mathbf{b}}-\mathbf{n}_{\mathbf{e}}\right)$ [measure $\mathrm{n}_{\mathrm{e}} / \mathrm{n}_{\mathrm{b}} \geq \mathrm{few} \%$ ]

\section{$\square$}

\section{Gridded collectors:}

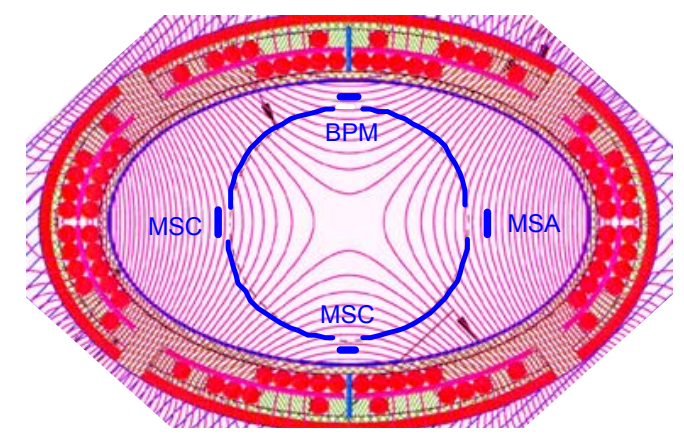

- Grids shield collectors from $\phi_{\mathbf{b}}\left(\sim 10^{3}\right.$ larger signal)

- Expelled ion current proportional to gas pressure in beam and electron generation rate $\mathbf{I}_{\mathbf{i}} \propto \mathbf{P}_{\mathbf{0}} \propto \mathbf{I}_{\text {e-trap }} \quad$ [measure $\mathrm{n}_{\mathrm{e}} / \mathrm{n}_{\mathrm{b}} \geq 0.1 \%$ ]

-Expelled ion energy is equal to beam potential at ion birth radius ' $r$ ', compare with simulations to $E_{i}=\phi_{b}(' r ')$,

- Detrapped electrons at end of beam pulse - if shielded from incident and scattered ions

- $\int I_{e} d t=$ total number trapped (should $=\int I_{e-t r a p} d t$ )

$-I_{e} V s \phi_{b}$ gives the depth of trapping 


\section{Electron emission from ion beam loss is sup- pressed by magnetic insulation of electrodes}

Diagnostics mounted on outside of beam-tube liner sample particle flux near axial center of quadrupole magnet

Beam

\section{beam-tube liner}

- Liner provides smooth surface facing beam

- Diagnostics can be assembled on tube, then easily installed in quadrupole magnet

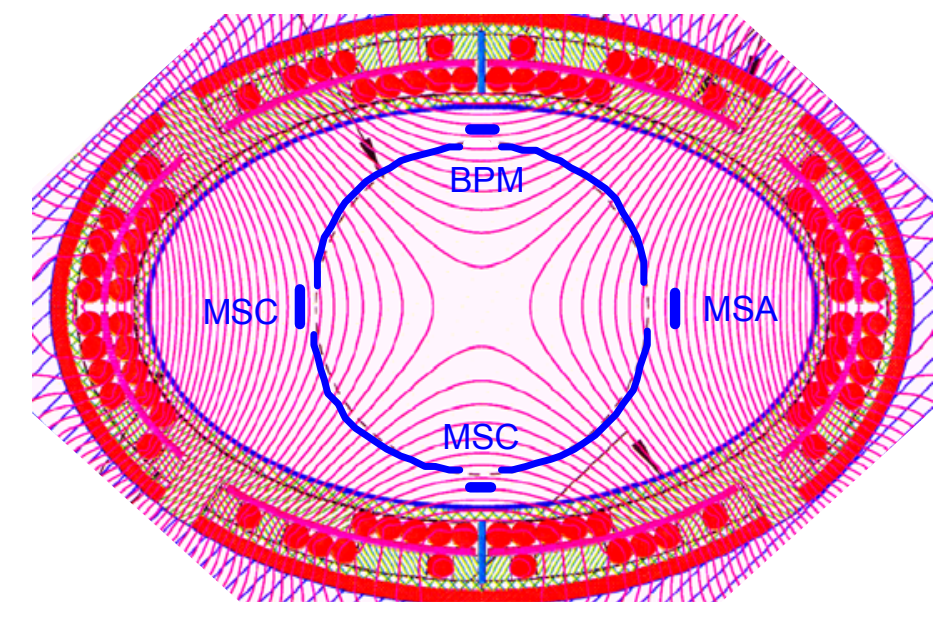

Beam into paper

BPM - Beam Potential (Position) Monitor capacitive pickup ( $\left.\sim 50 \mathrm{~A} / \mathrm{cm}^{2}\right)$ MSC - magnetically suppressed collector, grid(s) reduce capacitive pickup, measures expelled 'gas' ion current $\left(\sim 0.1 \mu \mathrm{A} / \mathrm{cm}^{2}\right.$ at $10^{-7}$ torr; $\mathrm{l}_{\mathrm{i}}=$ ionization rate $\propto$ pressure $\mathrm{P}(\mathrm{t})) \propto$ electron ionization rate $\left(\mathrm{I}_{\mathrm{e} \text {-trap }}\right)$

MSA - magnetically suppressed analyzer, like MSC but larger gap insulates $5 \mathrm{kV}$, measures expelled ion energy $=$ beam potential where ionized 


\section{Flush collectors run length of beam tube in one quadrupole}

\section{Collectors in quadrupole}

magnets

- Measure secondary electron current, infer beam loss, gas reflux, $n_{e}(t)$ and $n_{0}(t)$ [from GESD]

- Measure number and trappingenergy of electrons at end-ofpulse [Difficult, $\leq 1 \% \mathrm{I}_{\text {capacitive] }}$

- 2 collectors per quadrant determine beam loss. Collectors are effective wall.
4 collectors per quadrant (top) to compare loss at lobes Vs cusps

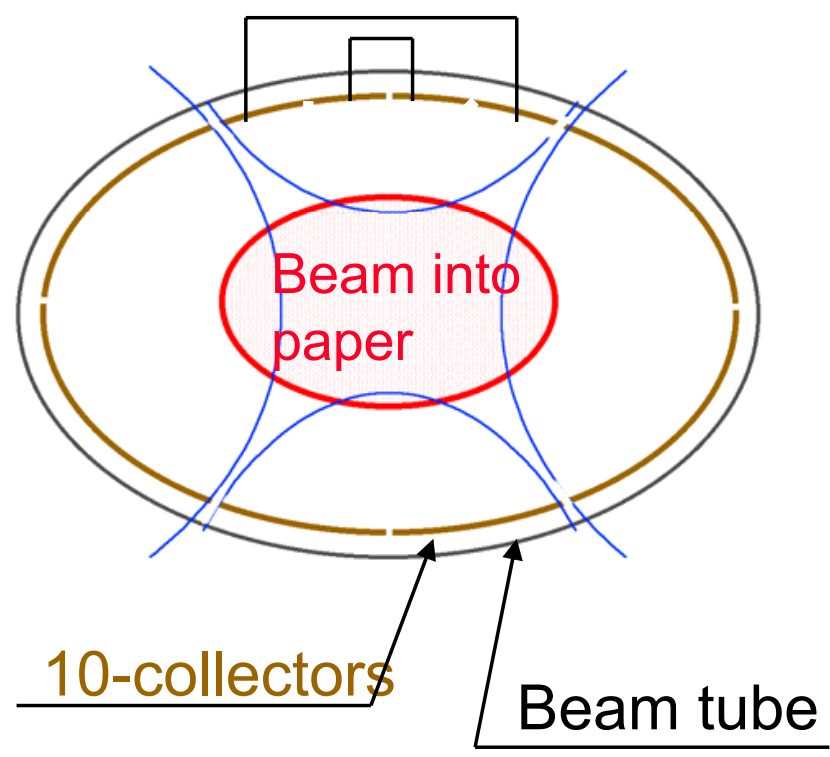




\section{Gas desorption and electron emission predicted to scale with ion stopping rate $\mathrm{dE} / \mathrm{dx}$}

- Gas desorption attributed to electronic energy loss of ions ${ }^{1}$ (which is the largest part of the total stopping power): scaling quadratically with $\mathrm{dE} / \mathrm{dx}$ at low temp, linearly with $\mathrm{dE} / \mathrm{dx}$ above $100 \mathrm{~K}$.

- Electron emission also scales linearly with electronic stopping power. ${ }^{2}$

1. W. L. Brown, et al., Phys. Rev. Letters 45, 1632 (1980).

2. P. Thieberger, et al., Phys. Rev. A 61, 042901-1 (2000).

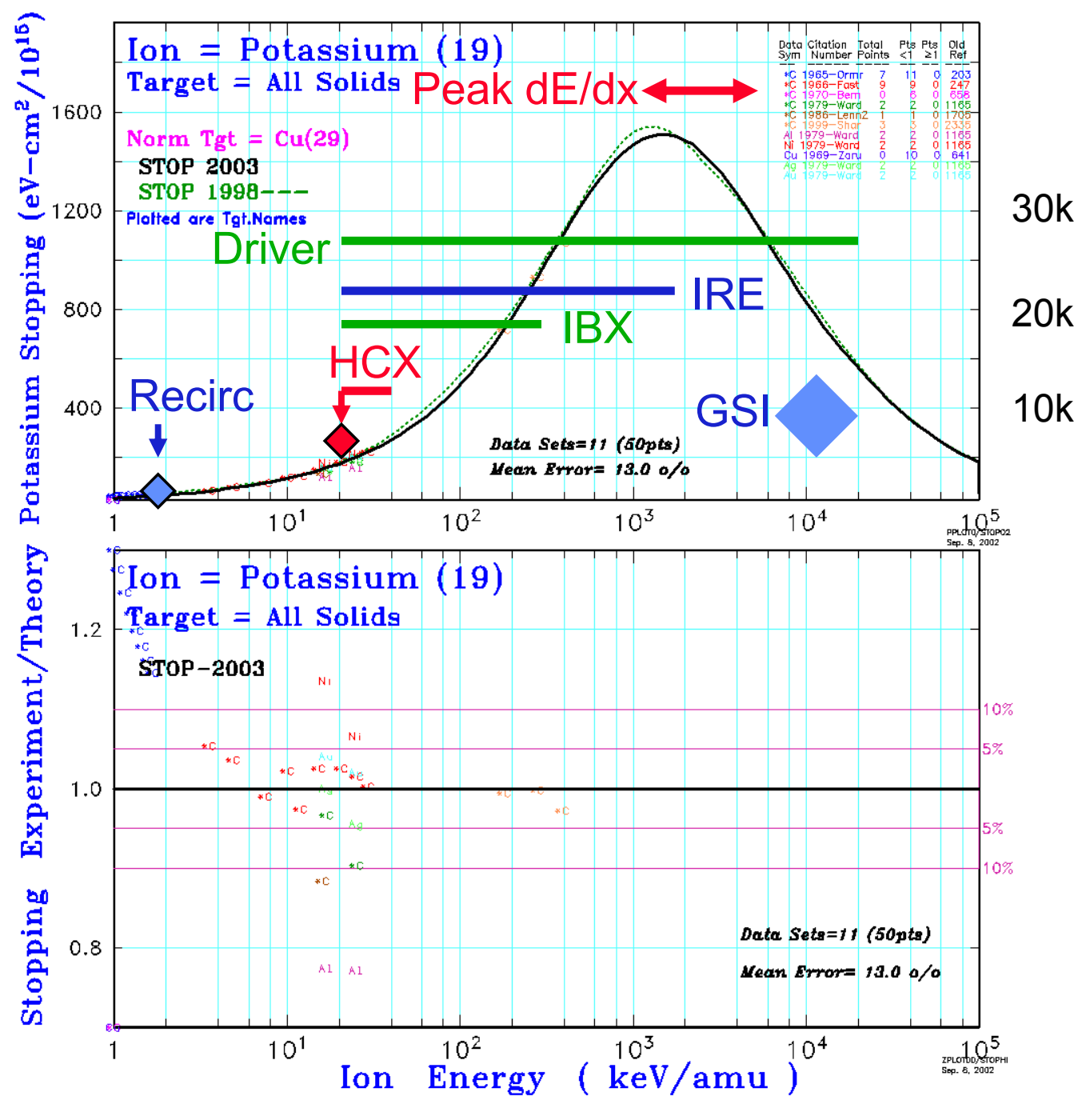




\section{We measure electron emission and gas desorption from $1 \mathrm{MeV} \mathrm{K}^{+}$beam impact on target}

\section{Gas, electron source diagnostic (GESD)}
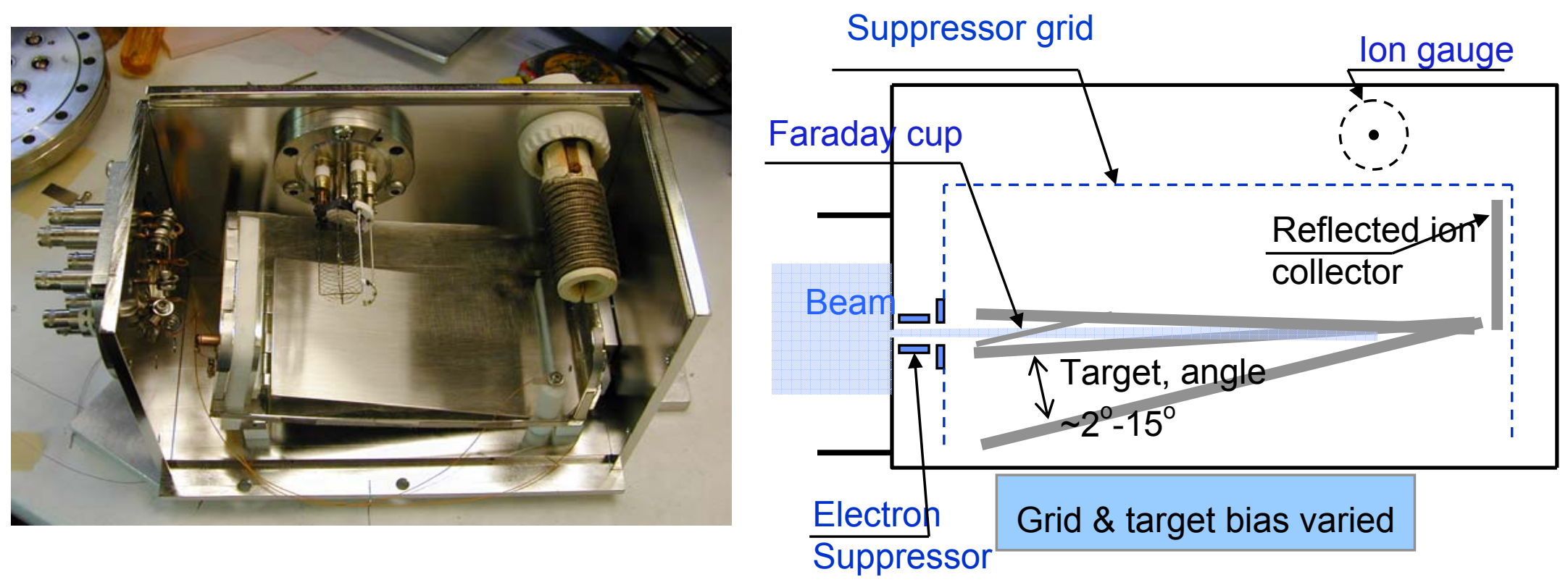

- Measure number \& energy of electrons and gas per incident $\mathrm{K}^{+}$ion

- Calibrate secondary electron measurements vs. beam loss

- Evaluate mitigation techniques: baking, cleaning, surface treatment... 


\section{Current-Voltage characteristics of GESD Faraday cup and target, indicate reliable current measurements}

- Negative Faraday cup measures beam current into GESD.

- Positive Faraday cup measures electrons from ionization of desorbed gas.

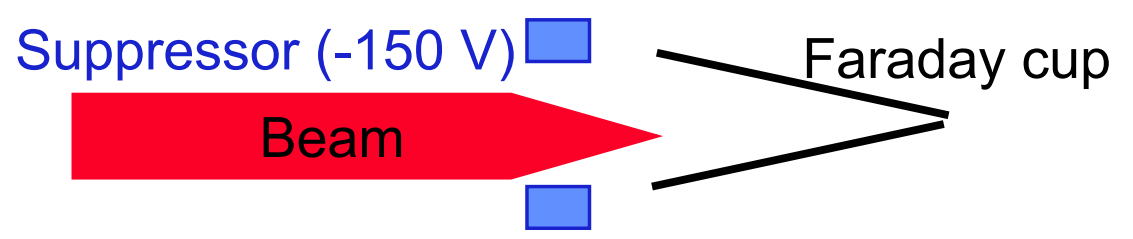

GESD Faraday Cup I-V Characteristic, Vs=-200 V, Vg=-150 V, Data
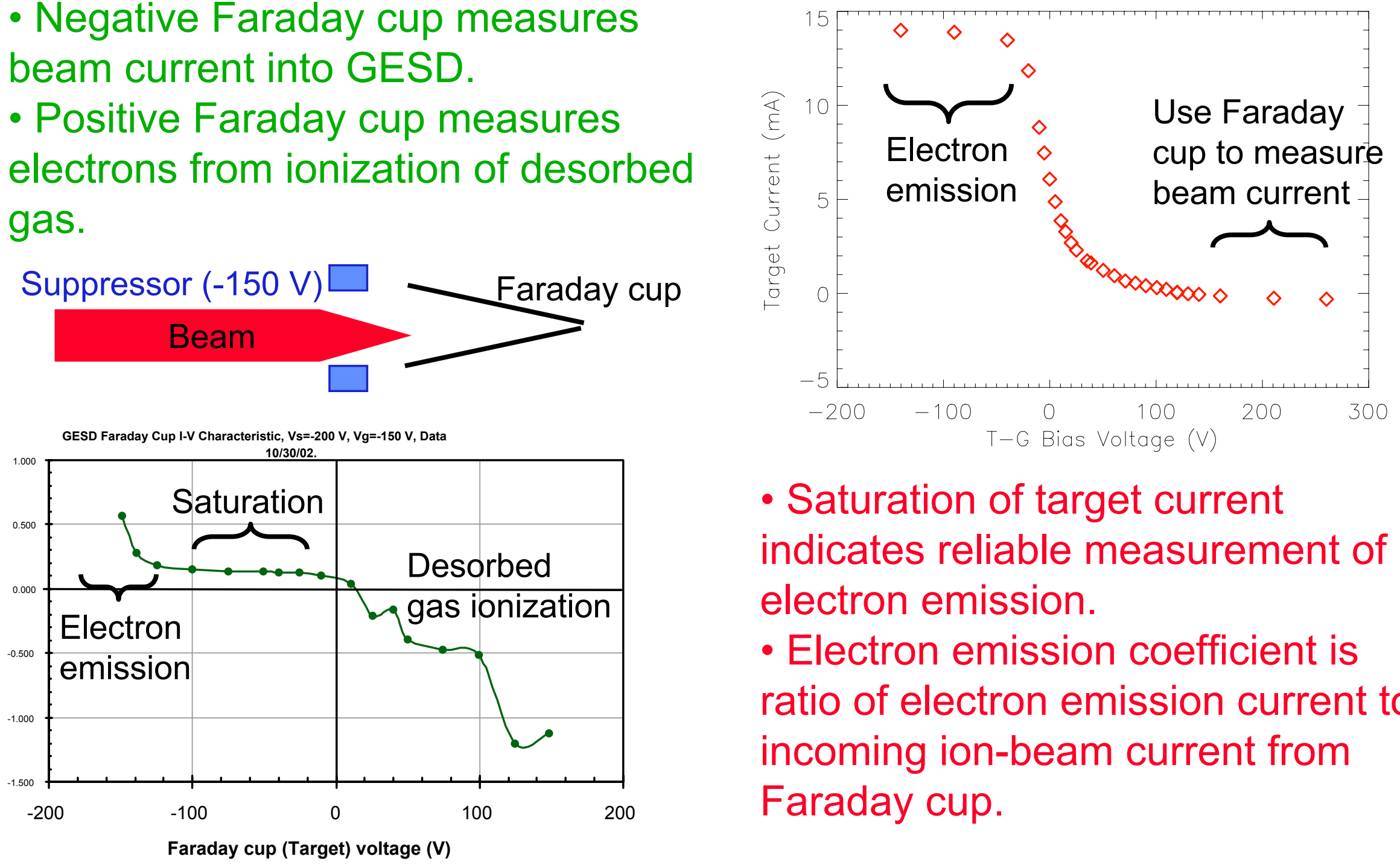

- Saturation of target current indicates reliable measurement of electron emission.

- Electron emission coefficient is ratio of electron emission current to incoming ion-beam current from Faraday cup. 


\section{GESD electron emission coefficient (EEC) varies with $\cos (\theta)^{-1}$}

- Simple model gives $\cos (\theta)^{-1}$

- Electrons from depth $>\delta$

$(\delta \sim 1 \mathrm{~nm})$ cannot leave surface

- lon path length in depth $\delta$ is L.

- Results depart from this near grazing incidence where the distance for nuclear scattering is $<\mathrm{L}^{1}$
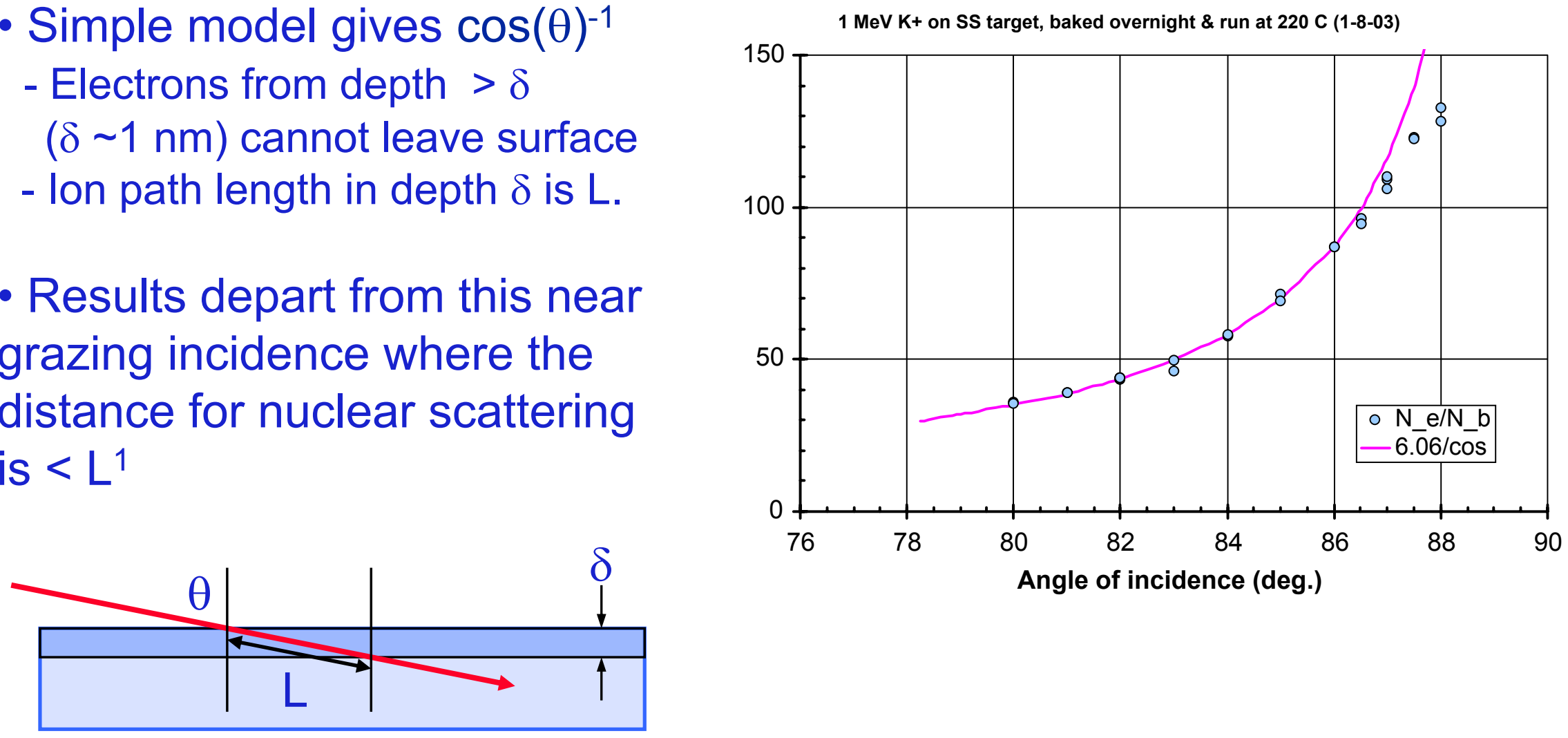

$$
\mathrm{L}=\delta / \cos (\theta)
$$

1. P. Thieberger,A. L. Hanson, D. B. Steski, et al., Phys. Rev. A 61, 42901 (2000). 


\section{GESD gas desorption coefficient varies with $\cos (\theta)^{-\alpha}, \alpha<1$}

Hypothesis:

- Gas desorption results from electronic sputtering of gas film on surface plus dust on surface.

- Film results in $\cos (\theta)^{-1}$.

- At grazing incidence on dust, some dust particles are partially or totally hidden behind others, if ion penetrates it is at reduce energy and $\mathrm{dE} / \mathrm{dx}$.

- Then dust desorption scales more slowly than $\cos (\theta)^{-1}$.

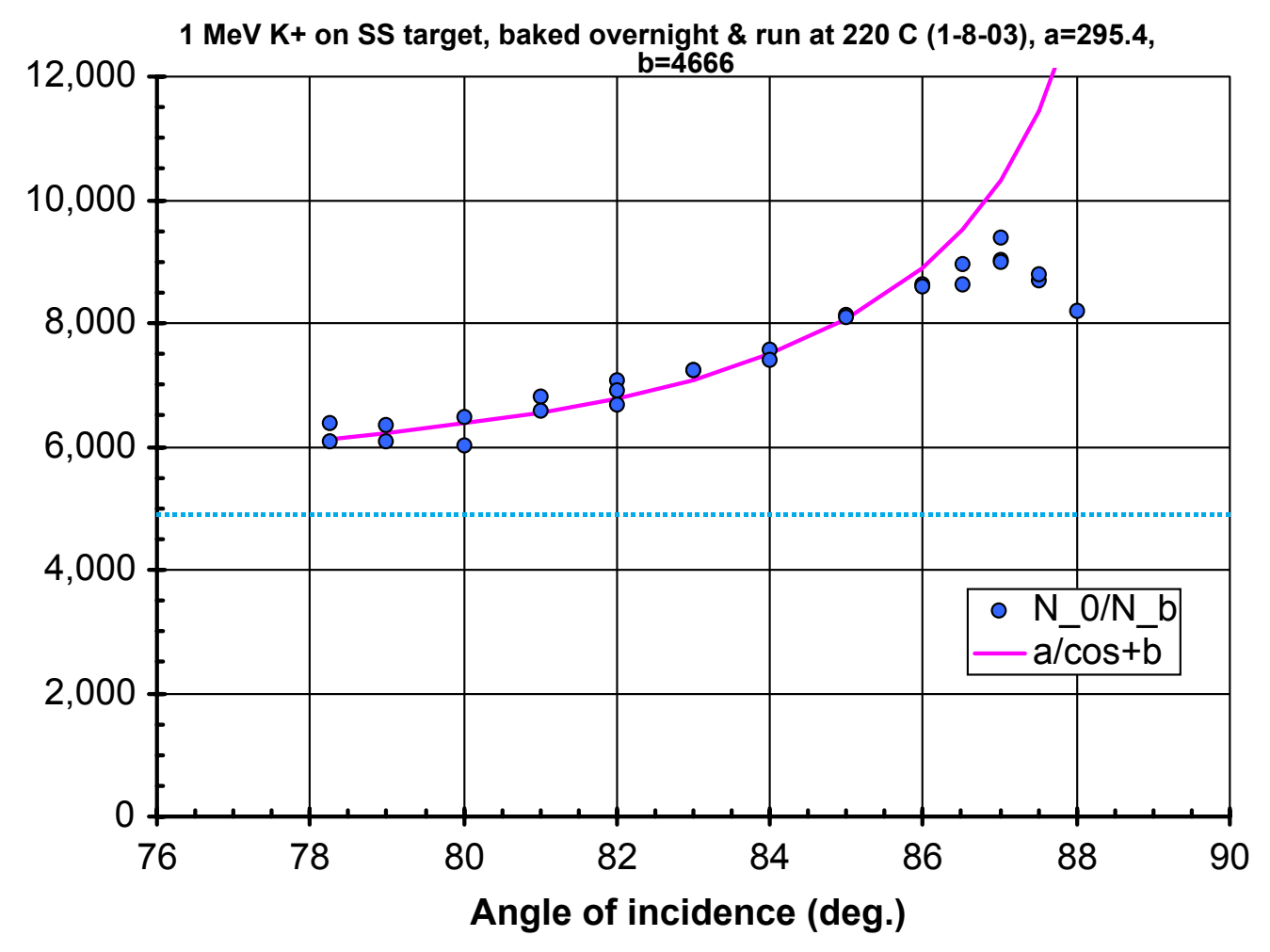

Beam

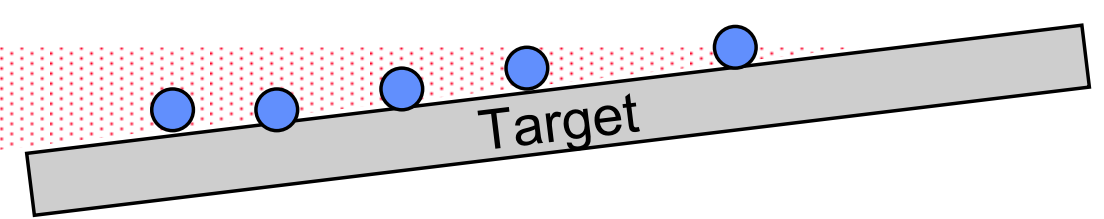




\section{Possible areas for collaboration}

\section{At GSI}

- Measure gas desorption and electron emission coefficients for ion energies in range of 1.4(?) to 20+ MeV/amu

\section{At HIF-VNL}

- GESD measure gas desorption \& electron emission Now coefficients for ions in range of 25-45 keV/amu

- Mitigation techniques: Cleaning

Scattered ion suppression

Halo scrape, electron trap

Now

FY03

?

- Trapping efficiency for secondary electrons

'03-04

- Detrapping electrons by induction cell 


\section{Summary}

\section{Overview}

- Electron clouds may establish "floor" on HIF driver costs

- Coordinated theory, computation, and experimental program initiated on electron effects in heavy-ion beams

\section{Experimental program}

- Electron emission coefficients scale with $\cos ^{-1}(\theta)$ for $1 \mathrm{MeV}$ $\mathrm{K}^{+}$incident on stainless-steel near grazing incidence.

- Gas desorption scales with $\cos ^{-\alpha}(\theta)$ for either $\alpha<<1$, or $\alpha \sim 1$ with zero offset. Also desorption $\propto \mathrm{dE} / \mathrm{dx}$ between 0.08 and $1 \mathrm{MeV}$.

- Hostile environment in quads evaluated, and an array of diagnostics constructed to measure electron parameters.

- Four magnetic quadrupoles with electron diagnostics ready to be installed in the High-Current Experiment (HCX). 